\title{
Neutrophil-Lymphocyte Ratio Predicts Organ Salvage in Testicular Torsion with Marginal Diagnostic Delay
}

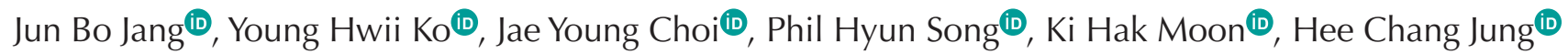 \\ Department of Urology, College of Medicine, Yeungnam University, Daegu, Korea
}

Purpose: Though prompt diagnosis to minimize symptom duration (SD) is highly associated with organ salvage in cases of testicular torsion (TT), SD is subjective and hard to determine. We thus investigated the clinical implications of systemic inflammatory responses (SIRs) as potential surrogates of SD to improve testis survival.

Materials and Methods: Sixty men with TT that underwent immediate operation among orchiectomy and orchiopexy following a visit to a single emergency department were retrospectively enrolled. Mandatory laboratory tests conducted included neutrophil, lymphocyte, and platelet counts.

Results: Mean age and SD was $15.7 \pm 3.7$ years and $8.27 \pm 4.98$ hours, respectively. Thirty-eight $(63.3 \%)$ underwent orchiectomy and the remaining 22 underwent orchiopexy. Leukocytosis $(\mathrm{p}=0.001)$ and neutrophil-lymphocyte ratio $(\mathrm{NLR}, \mathrm{p}<0.001)$ were significantly lower in the orchiopexy group as was SD $(3.27 \pm 1.88$ vs. $11.16 \pm 3.80, p<0.001)$. Although multivariate model showed that the only single variable associated with receipt of orchiopexy was $S D$ (odds ratio $[O R]=0.259, p<0.001$ ), it also revealed NLR as a sole SIR associated with SD ( $B=0.894, p<0.001)$. While $93.3 \%$ with a SD of within 3 hours underwent orchiopexy, only $26.6 \%$ of affected testes were preserved between 3 to 12 hours $(n=30)$. When multivariable analysis was applied to those with window period, NLR alone predicted orchiopexy rather than orchiectomy $(\mathrm{p}=0.034, \mathrm{OR}=0.635$, $\mathrm{p}=0.013)$. The area under curve between SD (0.882) and NLR (0.756) was similar $(\mathrm{p}=0.14)$.

Conclusions: This study showed NLR independently predicted testis survival by proper surgical correction particularly for patients with marginally delayed diagnosis, which suggest the clinical usefulness for identifying candidates for orchiopexy in emergency setting.

Keywords: Inflammation; Orchiectomy; Orchiopexy; Spermatic cord torsion; Symptom assessment

This is an Open Access article distributed under the terms of the Creative Commons Attribution Non-Commercial License (http://creativecommons.org/licenses/by-nc/4.0) which permits unrestricted non-commercial use, distribution, and reproduction in any medium, provided the original work is properly cited.

\section{INTRODUCTION}

Testicular torsion (TT), a rotation of the testis with twisting of the longitudinal axis of the spermatic cord, is a surgical emergency that requires prompt treat- ment to secure survival of the affected testis. Testicular salvage rates hinge on degree of torsion and the duration of subsequent ischemia. Delays in care may necessitate orchiectomy, which occurs in up to $31 \%$ to $41 \%$ of TT cases $[1,2]$ and can result in reduced fertility,

Received: Jun 10, 2018 Revised: Aug 28, 2018 Accepted: Aug 28, 2018 Published online Nov 5, 2018

Correspondence to: Young Hwii Ko iD https://orcid.org/0000-0002-9150-4292

Department of Urology, College of Medicine, Yeungnam University, 170 Hyeonchung-ro, Nam-gu, Daegu 42415, Korea.

Tel: +82-53-620-3695, Fax: +82-53-627-5535, E-mail: urokyh@naver.com 
testicular hormonal dysfunction [3] and psychological trauma [2]. As such, torsion is the third leading cause of malpractice lawsuits among teenage boys [4]. Regarding the proper management of TT, especially with respect to avoiding orchiectomy, it is well-known that time from symptom onset to surgical correction is the most important factor, and that prompt correction is associated with greater likelihood of organ salvage. More specifically, testicular recovery is more likely if intervention is conducted within 6 hours of symptom onset [5], and dramatically less likely after 12 hours [6].

However, time elapsed since symptom onset is wholly reliant on patient recall and is difficult to obtain with surety in young children, and pubescent males tend to be hesitant about seek medical attention for conditions involving their genitalia [7,8]. In addition, after arrival at an emergency department (ED), further evaluations on the potential cause of acute scrotal pain may cause additional delays, as well as transfer to another hospital [9,10]. Therefore, accurate selection for surgery should be implemented from the moment of ED arrival to minimize delays particularly for orchiopexy, a preventive procedure for the salvaged testis. As an attempt to identify such patients earlier using emergency setting blood sampling, we investigated the clinical implications of systemic inflammatory responses (SIRs) tested at time of admission as potential surrogates of symptom duration, and its association with testicular survival.

\section{MATERIALS AND METHODS}

\section{Patient enrollment and treatment decision- making}

Based on a retrospective chart review, consecutive men with TT that underwent immediate operation after visiting ED of Yeungnam University Medical Center between January 2005 and July 2015 were selectively enrolled, after obtaining institutional review board approval. At our institution, we made it mandatory in 2000 that all patients visiting our ED complaining of acute scrotal pain undergo routine ultrasonography and a physical examination by a urological specialist. Using these two modalities in combination, a clinical diagnosis of TT can be made preoperatively and an emergency operation immediately prepared when either modality produces a suspicious or positive finding. At the operation field, decisions regarding orchiectomy or orchiopexy were made by the primary surgeon following an assessment of testicular viability after relieving the twisted spermatic cord structure by applying warm saline for a minimum of 5 minutes.

\section{Variables obtained}

When patients visited our ED, they or their caregivers were questioned regarding symptom duration and responses were recorded by an employee not otherwise associated with this study. Symptom duration was defined as time between symptom onset (acute pain on scrotum) to arrival at our ED. After history-taking, it institutional policy to conducted mandatory laboratory tests that include white blood cell (WBC) count and differential neutrophil and lymphocyte counts, hemoglobin, platelets, and serum C-reactive protein level in order to expedite diagnosis. Neutrophil-lymphocyte ratio (NLR) and platelet-lymphocyte ratio (PLR) were calculated using these results. The time between visit to $\mathrm{ED}$ and beginning of anesthesia at operating room was also collected from the medical record.

\section{Statistical analysis}

Differences between the orchiectomy and orchiopexy groups were compared using the Mann-Whitney Utest and the chi-square test, and bivariate correlation analysis was used to identify associations between variables. A Logistic regression model was utilized to identify independent factors of orchiopexy. A multiple linear regression model was also used to identify associations between continuous variables. In order to suggest reliable cut-off values for variable, receiver operating characteristic (ROC) curve analysis was performed and areas under the curve were calculated then compared. The analysis was performed using IBM SPSS ver. 21.0 (IBM Co., Armonk, NY, USA) using twosided tests and a significance level of 5\%. ROC curves were compared using MedCalc version 18.2 (MedCalc Software, Ostend, Belgium).

\section{Ethics statement}

The present study was conducted as a consecutive case series, in which patients who received a similar treatment were followed and all outcomes were recorded and analyzed. Due to the nature of the diseases requiring urgent surgical treatment, the data was obtained by a reviewed medical record after permission of Institutional Review Board of Yeungnam University 
Medical Center (YUMC 2018-05-026).

\section{RESULTS}

\section{Patients characteristics}

Sixty men of mean age, $15.7 \pm 3.7$ years were recruited. Thirty-eight patients (63.3\%) underwent orchiectomy and the others $(n=22,36.3 \%)$ underwent orchiopexy. Patient characteristics are summarized in Table 1. The mean time from symptom onset to ED arrival was $8.27 \pm 4.98$ hours (range, $1-18$ hours). The mean time from ED arrival to operating room was $116.7 \pm 25.9$ minutes, which was similar across orchiopexy and orchiectomy groups $(\mathrm{p}=0.988)$. WBC $(8,943 \pm 2,428 v s$. $10,871 \pm 3,224, \mathrm{p}=0.01)$ and NLR $(2.72 \pm 1.33$ vs. $4.81 \pm 3.09$, $\mathrm{p}<0.001$ ) were significantly lower in the orchiopexy group as was mean time from symptom onset to ED arrival $(3.27 \pm 1.88$ vs. $11.16 \pm 3.80, \mathrm{p}<0.001)$.

\section{Predictor for orchiopexy}

Although univariate analysis indicated WBC $(\mathrm{p}=0.026)$, NLR $(\mathrm{p}=0.009)$, and PLR $(\mathrm{p}=0.049)$ were associated with receipt of orchiopexy (Table 2), the multivariate model showed that the only single variable associated with receipt of orchiopexy was symptom duration (odds ratio $[\mathrm{OR}]=0.259$, enter method, $\mathrm{R}^{2}=0.833$, $\mathrm{p}=0.014)$. However, multiple linear regression analysis revealed that NLR was solely correlated with symptom duration among SIRs $(B=0.894, p<0.001$, forward stepwise method, $R^{2}=0.244, p<0.001$ ). In a simple correlation analysis, a moderate association between them was revealed $\left(r=0.391, p=0.002, R^{2}=0.251 ;\right.$ Fig. 1$)$.

Table 1. Patient characteristics

\begin{tabular}{|c|c|c|c|c|}
\hline Characteristic & Total $(n=60)$ & Orchiopexy $(n=22)$ & Orchiectomy $(n=38)$ & p-value \\
\hline Age (y) & $14.73 \pm 2.48$ & $14.64 \pm 2.01$ & $14.79 \pm 2.74$ & 0.81 \\
\hline $\mathrm{Hb}(\mathrm{g} / \mathrm{dL})$ & $14.56 \pm 1.04$ & $14.49 \pm 0.98$ & $14.61 \pm 1.08$ & 0.68 \\
\hline WBC $(/ \mu \mathrm{L})$ & $10,164 \pm 3,081$ & $8,943 \pm 2,428$ & $10,871 \pm 3,224$ & 0.01 \\
\hline Neutrophil (\%) & $67.15 \pm 14.55$ & $63.82 \pm 8.87$ & $69.08 \pm 16.81$ & 0.12 \\
\hline Lymphocyte (\%) & $23.75 \pm 12.33$ & $26.15 \pm 6.07$ & $22.36 \pm 14.70$ & 0.17 \\
\hline Platelet $(/ \mu \mathrm{L})$ & $272,833 \pm 56,691$ & $261,818 \pm 62,545$ & $279,210 \pm 52,827$ & 0.28 \\
\hline $\mathrm{CRP}(\mathrm{mg} / \mathrm{dL})$ & $1.08 \pm 1.26$ & $0.97 \pm 1.16$ & $1.15 \pm 1.33$ & 0.59 \\
\hline NLR & $4.04 \pm 2.76$ & $2.72 \pm 1.33$ & $4.81 \pm 3.09$ & $<0.001$ \\
\hline PLR & $148.2 \pm 75.4$ & $121.9 \pm 39.7$ & $163.4 \pm 86.7$ & 0.01 \\
\hline Symptom duration (h) & $8.27 \pm 4.98$ & $3.27 \pm 1.88$ & $11.16 \pm 3.80$ & $<0.001$ \\
\hline ED arrival to OR time (min) & $116.7 \pm 25.9$ & $116.6 \pm 32.3$ & $116.7 \pm 21.9$ & 0.988 \\
\hline
\end{tabular}

Values are presented as mean \pm standard deviation.

Hb: hemoglobin, WBC: white blood cell, CRP: C-reactive protein, NLR: neutrophil-lymphocyte ratio, PLR: platelet-lymphocyte ratio, ED: emergency department, OR: operating room.

Table 2. A summary of univariate and multivariate analysis results

\begin{tabular}{|c|c|c|c|c|c|c|c|c|}
\hline \multirow{3}{*}{ Variable } & \multicolumn{4}{|c|}{ Univariate } & \multicolumn{4}{|c|}{ Multivariate } \\
\hline & \multirow{2}{*}{ p-value } & \multirow{2}{*}{ OR } & \multicolumn{2}{|c|}{$95 \% \mathrm{Cl}$} & \multirow{2}{*}{ p-value } & \multirow{2}{*}{ OR } & \multicolumn{2}{|c|}{$95 \% \mathrm{Cl}$} \\
\hline & & & Lower & Upper & & & Lower & Upper \\
\hline Age & 0.817 & 0.975 & 0.788 & 1.207 & 0.502 & 0.838 & 0.5 & 1.404 \\
\hline $\mathrm{Hb}$ & 0.68 & 0.898 & 0.537 & 1.499 & 0.43 & 1.759 & 0.433 & 7.154 \\
\hline WBC & 0.026 & 1 & 1 & 1 & 0.74 & 1 & 0.998 & 1.001 \\
\hline Platelet & 0.253 & 1 & 1 & 1 & 0.554 & 1 & 1 & 1 \\
\hline CRP & 0.591 & 0.888 & 0.575 & 1.371 & 0.544 & 0.772 & 0.335 & 1.78 \\
\hline NLR & 0.009 & 0.701 & 0.539 & 0.914 & 0.955 & 1.096 & 0.047 & 25.676 \\
\hline PLR & 0.049 & 0.991 & 0.982 & 1 & 0.647 & 1.026 & 0.919 & 1.146 \\
\hline Symptom duration & $<0.001$ & 0.443 & 0.28 & 0.7 & 0.014 & 0.259 & 0.088 & 0.764 \\
\hline
\end{tabular}

OR: odds ratio, Cl: confidence interval, Hb: hemoglobin, WBC: white blood cell, CRP: C-reactive protein, NLR: neutrophil-lymphocyte ratio, PLR: platelet-lymphocyte ratio. 


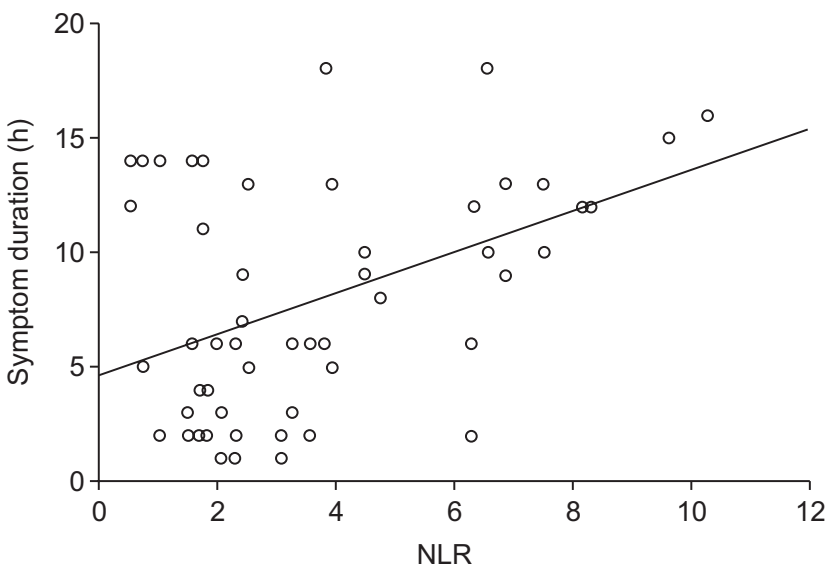

Fig. 1. The correlation between symptom duration and neutrophillymphocyte ratio (NLR).

Table 3. Stratification of patients by symptom duration to the surgical outcomes

\begin{tabular}{lccc}
\hline & Orchiectomy & Orchiopexy & Total \\
\hline Within 3 hours & $1(6.7)$ & $14(93.3)$ & $15(25.0)$ \\
3 to 6 hours & $5(45.5)$ & $6(54.5)$ & $11(18.3)$ \\
6 to 12 hours & $17(89.5)$ & $2(10.5)$ & $19(31.7)$ \\
12 to 18 hours & $15(100)$ & $0(0)$ & $15(25.0)$ \\
Total & $38(63.3)$ & $22(36.7)$ & $60(100)$ \\
\hline
\end{tabular}

Values are presented as number (\%).

\section{Adjusting symptom duration by stratification}

When the 60 study subjects were divided based on symptom duration, we found the majority with a symptom duration of $<3$ hours underwent orchiopexy (93.3\%) and that no patient with a symptom duration of $>12$ hours underwent orchiopexy (Table $3 ; \mathrm{p}<0.001$ ). With symptom durations of between 3 to 12 hours ( $n=30$, $50.0 \%$ ), affected testes were preserved in only $26.6 \%$ of those. When multivariable analysis was applied to these 30 patients with 'window period', NLR alone predicted orchiopexy rather than orchiectomy $(\mathrm{p}=0.034$, $\mathrm{OR}=0.635$, 95\% confidence interval: 0.417-0.966, forward stepwise method, $R^{2}=0.236, p=0.013$ ). When $R O C$ analysis was applied, the area under the curve (AUC) of symptom duration was $0.882(\mathrm{p}<0.001$, sensitivity $77.3 \%$ and specificity $91.8 \%$ at a cut-off of 6.5 hours) and that of NLR was 0.756 ( $\mathrm{p}=0.018$, sensitivity $81.8 \%$ and specificity $63.6 \%$ at a cut-off of 2.3), and these AUCs were not significantly different ( $\mathrm{p}=0.14$; Fig. 2 ).

\section{DISCUSSION}

TT is a surgical emergency that occurs in 1 in 4,000

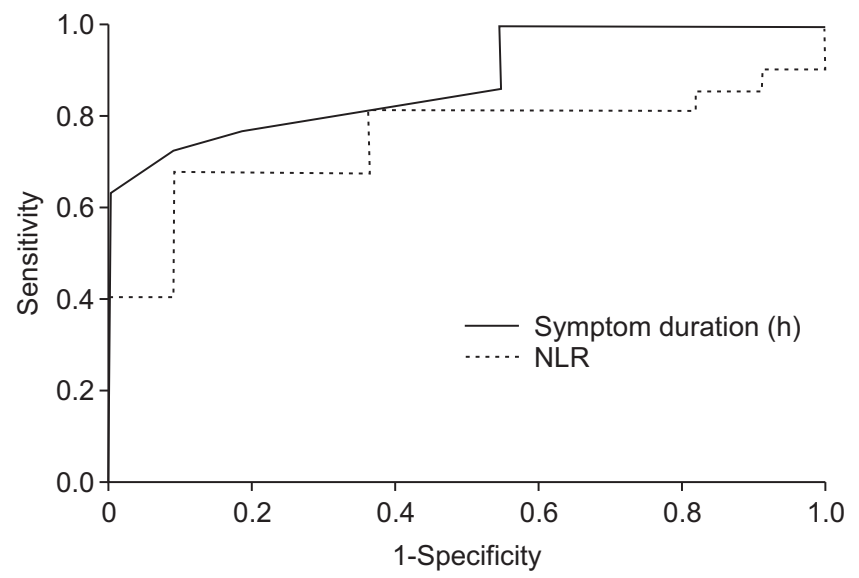

Fig. 2. Receiver operating characteristic curve comparing symptom duration and neutrophil-lymphocyte ratio (NLR) in 33 men with symptom duration within 3 to 12 hours.

males younger than 25 years old, and its diagnosis in the ED setting remains challenging for urologists [11]. Testicular salvage for patients with TT requires that three conditions be met, that is, timely presentation, rapid diagnosis, and curative intervention [12]. In order to increase the rate of testicular survival by minimizing the time taken for diagnosis, we focused on SIRs based on their reported benefits for the identification of malignant conditions. To the best of our knowledge, this is the first study to evaluate the use of SIRs as indicators for orchiopexy in TT patients. Several interesting findings were obtained from this series.

Multivariate analysis for predictors of orchiopexy showed that symptom duration was the only significant predictor, which concurs with previous reports. Given the objective nature of symptom duration and the vague symptoms of TT, which include nausea and/ or vomiting, particularly in young patients [6], we searched for relations between tests routinely performed at time of ED visit and symptom duration. Of the blood tests performed, multivariate analysis showed that only NLR was significantly associated with symptom duration. Patients were stratified by symptom duration, and because it is known that symptom durations of $<3$ hours or $>12$ hours tend to result in orchiopexy or orchiectomy, respectively, we focused on 33 patients with symptom durations of 3 to 12 hours. In this patient group, NLR was the only single predictor of orchiopexy and had an AUC similar to that of symptom duration. These findings indicate NLR is a potential indicator of testis viability in TT, especially for patients with a symptom duration marginally be- 
yond the 'golden time' for affected testis salvage.

In an emergency setting, SIRs have been studied as potential indicators of testicular status, and two reports concluded that NLR reliably differentiates TT and epididymitis. Güneş et al [13] investigated various SIRs in a cohort of 75 TT patients and 56 age-matched healthy controls, and reported significant relationships between scrotal tenderness and NLR, PLR, and platelet count, and that only NLR significantly differentiated between those with short $(<12$ hours) and long (>12 hours) symptom durations. However, besides lack of multivariable approach and uneven distribution of each group, TT was diagnosed based on physical examination by a urological resident without radiologic evidence in their study, and no information was provided on testes survival after diagnosing TT.

Because neutrophils play a crucial role in the inflammatory process [14], Bitkin et al [15] recently evaluated NLR and other SIRs to determine whether they could aid the differentiation of TT and epididymitis. They enrolled 51 patients that underwent surgery for TT, 50 patients treated for epididymitis, and 52 healthy individuals. SIRs were found to display statistically different distributions for the two disease entities, and they reported NRL identified TT with an AUC of 0.744 and a sensitivity and specificity of $70.1 \%$ and $76.9 \%$, respectively. However, this study was based on simple group comparisons, and the predictive powers of SIRs were not tested using a multivariate approach.

Several limitations of the present study warrant consideration. First, because of its retrospective nature, which is unavoidable for disease entities requiring emergency surgical treatment, physical examinations were performed by different physicians and ultrasonography was performed by different radiologists. Also, orchiopexy and orchiectomy were performed by several surgeons with different surgical volumes and experience of TT. In addition, the degree of cord twisting, which effects on the survival of testis was not collected from lack of record. However, quite similar time from ED arrival to operating room regardless of testicular survival which relatively shorter than other report [16] reflect the quality of surgical management in this series. Second, while our results indicate the usefulness of NRL as a surrogate of symptom duration, the accuracy of the suggested cut-off was unavoidably affected by the relatively small patients number recruited and the single center nature of the study. Furthermore, we excluded etiologies of acute scrotum, such as, epididymitis or torsion of appendix testis, which overlap with TT clinically and in terms of laboratory findings. In addition, our NLR cut-off of 2.3 is lower than those previously reported (2.5 [13] and 2.95 [15]). On the other hand, in the present study diagnosis was confirmed in the most reliable way by surgical exploration. Though the findings of the present study require confirmation by other researchers, the notion that NLR may be clinically useful for identifying TT patients suitable for organ salvage is appealing given the rapidity of disease progression.

\section{CONCLUSIONS}

Though symptom duration was found to be the most reliable indicator of testicular viability at time of surgery, this study also showed NLR independently predicted testis survival by proper surgical correction particularly for patients with marginally delayed diagnosis, which suggest the potential clinical usefulness of the novel, readily accessible variable for identifying candidates for emergency orchiopexy rather than orchiectomy.

\section{ACKNOWLEDGEMENTS}

This work was supported by a Yeungnam University Research Grant (2017).

\section{Disclosure}

The authors have no potential conflicts of interest to disclose.

\section{Author Contribution}

Drafting and basic concept: Ko YH, Song PH. Data collection: Song PH, Moon KH, Jung HC. Statistical analysis: Ko YH, Choi JY. Manuscript editing: Jang JB, Choi JY.

\section{REFERENCES}

1. Zhao LC, Lautz TB, Meeks JJ, Maizels M. Pediatric testicular torsion epidemiology using a national database: incidence, risk of orchiectomy and possible measures toward improving the quality of care. J Urol 2011;186:2009-13.

2. Bodiwala D, Summerton DJ, Terry TR. Testicular prostheses: development and modern usage. Ann R Coll Surg Engl 2007; 
89:349-53.

3. Fisch H, Laor E, Reid RE, Tolia BM, Freed SZ. Gonadal dysfunction after testicular torsion: luteinizing hormone and follicle-stimulating hormone response to gonadotropin releasing hormone. J Urol 1988;139:961-4.

4. Selbst SM, Friedman MJ, Singh SB. Epidemiology and etiology of malpractice lawsuits involving children in US emergency departments and urgent care centers. Pediatr Emerg Care 2005;21:165-9.

5. Bowlin PR, Gatti JM, Murphy JP. Pediatric testicular torsion. Surg Clin North Am 2017;97:161-72.

6. Boettcher M, Bergholz R, Krebs TF, Wenke K, Aronson DC. Clinical predictors of testicular torsion in children. Urology 2012;79:670-4.

7. Rampaul MS, Hosking SW. Testicular torsion: most delay occurs outside hospital. Ann R Coll Surg Engl 1998;80:169-72.

8. Nasrallah P, Nair G, Congeni J, Bennett CL, McMahon D. Testicular health awareness in pubertal males. J Urol 2000; 164:1115-7.

9. Bayne AP, Madden-Fuentes RJ, Jones EA, Cisek LJ, Gonzales ET, Reavis KM, et al. Factors associated with delayed treatment of acute testicular torsion-do demographics or interhospital transfer matter? J Urol 2010;184(4 Suppl):1743-7.

10. Yiee JH, Chang L, Kaplan A, Kwan L, Chung PJ, Litwin MS.
Patterns of care in testicular torsion: influence of hospital transfer on testicular outcomes. J Pediatr Urol 2013;9:713-20.

11. Barada JH, Weingarten JL, Cromie WJ. Testicular salvage and age-related delay in the presentation of testicular torsion. J Urol 1989;142:746-8.

12. Mansbach JM, Forbes P, Peters C. Testicular torsion and risk factors for orchiectomy. Arch Pediatr Adolesc Med 2005;159: 1167-71.

13. Güneş $M$, Umul M, Altok $M$, Akyuz $M$, İşoğlu CS, Uruc F, et al. Predictive role of hematologic parameters in testicular torsion. Korean J Urol 2015;56:324-9.

14. Kolaczkowska E, Kubes P. Neutrophil recruitment and function in health and inflammation. Nat Rev Immunol 2013;13: 159-75.

15. Bitkin A, Aydın M, Özgür BC, Irkilata L, Akgunes E, Keles $\mathrm{M}$, et al. Can haematologic parameters be used for differential diagnosis of testicular torsion and epididymitis? Andrologia 2018;50. doi: 10.1111/and.12819.

16. Afsarlar CE, Ryan SL, Donel E, Baccam TH, Jones B, Chandwani B, et al. Standardized process to improve patient flow from the emergency room to the operating room for pediatric patients with testicular torsion. J Pediatr Urol 2016;12:233.e14. 